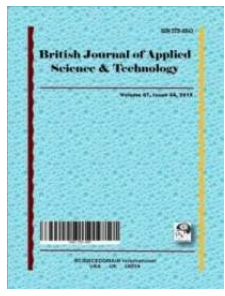

\title{
A Comparative Study of the Glycating Power of Simple Carbohydrates in the Maillard Reaction by Means of Conceptual DFT Descriptors
}

\author{
Juan Frau ${ }^{1}$ and Daniel Glossman-Mitnik ${ }^{1,2^{\star}}$ \\ ${ }^{1}$ Departamento de Química, Universitat de les Illes Balears, 07122 Palma de Mallorca, Spain. \\ ${ }^{2}$ Departamento de Medio Ambiente y Energía, Laboratorio Virtual NANOCOSMOS, Centro de \\ Investigación en Materiales Avanzados, Chihuahua Chih 31136, Mexico.
}

\section{Authors' contributions}

This work was carried out in collaboration between both authors. Author DGM conceived and designed the research and headed, wrote and revised while author JF contributed to the calculations and to the writing and the revision of the article. Both authors read and approved the final manuscript.

Article Information

DOI: 10.9734/BJAST/2017/32795

Editor(s):

(1) Robson Xavier Faria, Laboratory of Cell Communication, Oswaldo Cruz Institute, Oswaldo Cruz Foundation, Brazil. (2) Ya-mei Gao, College of life science and technology, Heilongjiang Bayi Agariculture University, Daqing, Heilongjiang, China. (3) Ming-Chih Shih, Department of Health and Nutrition Science, Chinese Culture University, Taiwan. (4) Meng Ma, Anhui University, Hefei, Anhui, China and Icahn Institute for Genomics and Multiscale Biology, Icahn School of Medicine at Mount Sinai, New York, USA Reviewers:

(1) Mohsen Kerkeni, Higher Institute of Biotechnology, Tunisia. (2) S. Simonetti, Universidad Nacional del Sur (UNS), Argentina. Complete Peer review History: http://www.sciencedomain.org/review-history/19110

Original Research Article

Received $16^{\text {th }}$ March 2017

Accepted $14^{\text {th }}$ May 2017

Published $17^{\text {th }}$ May 2017

\begin{abstract}
This study features an assessment of multiple density functionals in calculating the molecular structures and inherent properties in simple carbohydrates participating in nonenzymatic glycation involving amino acids and proteins in Maillard reactions. Calculations of chemical reactivity descriptors is attained in each molecular system using Conceptual DFT. A cross-sectional comparison of results obtained through $\triangle \mathrm{SCF}$ procedure is attained to check for accuracy and validity of the density functional in "Koopmans in DFT" (KID) procedure. The Fukui function indices and condensed dual descriptor $\Delta f(r)$ are used to examine the active sites where nucleophilic and electrophilic attacks occur. The assessment identifies several relationships involving glycating power and reactivity descriptors. The latter are found capable for predicting glycating behavior in complex carbohydrates.
\end{abstract}


Keywords: Computational chemistry; molecular modeling; carbohydrates; maillard reaction; conceptual DFT; chemical reactivity theory.

\section{INTRODUCTION}

The occurrences of nucleophilic addition involving nonenzymatic glycation initiate protein glycation reaction involving a carbonyl group derived from reeducating sugars and free amino group forming a reversible Schiff base. Progressive reactions of the glycated proteins produce complex structures identified as advanced glycation endproducts (AGEs) [1]. The AGEs formed have potential to initiate oxidative stress causing positive feedback loop. This in turn enhances the oxidative destruction of the brain. This explains the role of AGEs in causing neurodegenerative diseases and Alzheimer disease [2]. Besides, the formation of AGEs facilitates chronic diabetic complications [3]. These cases make it important to understand how various carbohydrates, amino acids and proteins react to facilitate mechanisms of such nature. Experiments have indeed demonstrated the interaction abilityin various carbonyl group molecules with amino groups identified as glycating power strongly influenced by their electronic properties and molecular structures.

Adrover et al. interesting study sought examining the kinetics involved in the interaction of hexoses and pentoses with pyridoxamine - known to inhibit formation of AGEs [4]. Their results revealed strong correlation in constant rates of reaction between the carbonyl group in all carbohydrates, pyridoxamine amino group and net NBO charges involving the carbonyl $\mathrm{C}$ atom linked with either hexose or pentose. The results suggested a possibility if quantifying the glycation power using the electronic properties of a specific molecule of reducing carbonyl. This provided an opportunity to build enhanced scale to predict glycation power of carbohydrates with more complex structures.

Both empirical and practical perspective emphasize the importance of complying with procedural assignment of $\mathrm{KS}$ HOMO opposite and identical to the vertical ionization potential, $\varepsilon_{H}=-I$. The same perspective applies in KS LUMO assigned equivalent and reverse of vertical electron affinity $\varepsilon_{L}=-A$. Besides this understanding, this empirical procedure coins KID representing "Koopmans in DFT". Furthermore, it implies the possibility of estimating whether density functional behavior comply with the KID procedure. This consideration is essential in calculating the Conceptual DFT descriptors integral in predicting and explaining the chemical reactivity of different molecular systems. Consequently, this study desires fulfilling two objectives. Firstly, it seeks conducting comparative examination of the behavior of multiple density functional including those drawn from the Minnesota family in describing the chemical reactivity of hexoses and pentoses molecular system captured in Fig. 1 below. Secondly, it seeks building enhanced glycation power scale using experimental rate constants derived from initial reaction between carbohydrates and amino groups. The empirical procedure will embrace Conceptual DFT descriptors calculated accurately.

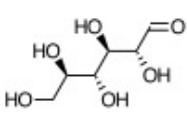

(a)

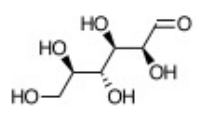

(b)

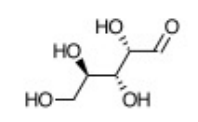

(c)

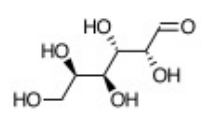

(d)<smiles>O=CC(O)C(O)C(O)CO</smiles>

(e)

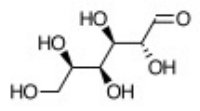

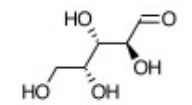

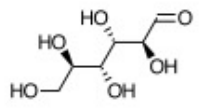

(g) (h)

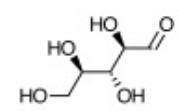

(i)

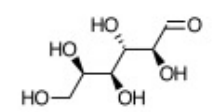

(j)

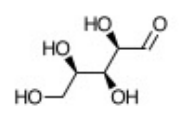

(k)

Fig. 1. Molecular structures of a) Allose, b) Altrose, c) Arabinose, d) Galactose, e) Glucose, f) Gulose, g) Lyxose, h) Mannose, i) Ribose, j) Talose and k) Xylose 
This study will feature some details and similar objectives in the Adrover et al study since they are yet to complete the examination of glycation between amino acids and protein through reducing carbonyls project [5]. While the study will publish such objectives and details, its present focus involves simple monosaccharides of aldoses form. Specifically, the study will examine the hexoses and pentoses leaving disaccharides and reducing carbonyls of ketoses form in the subsequent research. As earlier mentioned, this study will include multiple density functionals in providing a strong testing channel for the proposed KID procedure.

\section{THEORETICAL BACKGROUND}

This section features a similar theoretical background to previous researches [5-12] since the study is still an ongoing project. It inclusion serves to complete the structure of this in this stud, thereby retains the details described in those researches [5-12]. The chemical potential, $\mu$ is captured within the conceptual DFT framework as: [13-14] $\mu=(\partial E / \partial N)_{v(r)}=-\chi$, with $\chi$ representing the electronegativity. $\mathrm{A}$ similar approach is applied for global hardness $\eta$ defined as $\eta=\left(\partial^{2} E / \partial N^{2}\right)_{v(r)}$. In addition, this study applies the finite difference approximation for the chemical potential and global hardness as $\eta=(I-A) \approx\left(\varepsilon_{L}-\epsilon_{H}\right)=\eta_{K} . \varepsilon_{H}$ and $\varepsilon_{L}$ being the energies of the highest occupied molecular orbital, (HOMO) and lowest unoccupied molecular orbital (LUMO). The electrophilicity index, is again defined as [15] $\omega=\mu^{2} / 2 \eta=$ $(I+A)^{2} / 4(I-A) \approx\left(\epsilon_{L}+\epsilon_{H}\right)^{2} / 4\left(\varepsilon_{L}-\varepsilon_{H}\right)=\omega_{K}$, while the electrodonating $\omega^{-}$and electroaccepting $\omega^{+}$powers are well represented as [16] $\omega^{-}=$ $(3 I+A)^{2} / 16(I-A) \approx\left(3 \varepsilon_{H}+\varepsilon_{L}\right)^{2} / 16 \eta_{K}=\omega_{K}^{-}$and $\omega^{+}=(I+3 A)^{2} / 16(I-A) \approx\left(\varepsilon_{H}+3 \varepsilon_{L}\right)^{2} / 16 \eta_{K}=\omega_{K}^{+}, \mathrm{r}$ espectively. The definition emphasizes that larger $\omega^{+}$shows enhanced capability to accept charge and smaller $\omega^{-}$values indicating better electron donation. The following definition of net electrophilicity has been proposed as [17] $\Delta \omega^{ \pm}=$ $\omega^{+}-\left(-\omega^{-}\right)=\omega^{+}+\omega^{-} \approx \omega_{K}^{+}-\left(-\omega_{K}^{-}\right)=\omega_{K}^{+}+$ $\omega_{K}^{-}=\Delta \omega_{K}^{ \pm}$, as a comparison of the electroaccepting $\omega^{+}$and electrodonating $\omega^{-}$ powers.

The function $f(r)$ illustrates a reflection of the ability of molecular sites accepting or donating electrons defined using derivatives of $\rho(r)$ with reference to $N$, shown as $f(r)=$ $(\partial \rho(r) / \partial N)_{v(r)} . \rho(r$ derivatives on the right and left arise since the electrons number $N$ constitutes a discrete variable [18]. Further use of finite difference approximation in the above equation produces a twofold definition of Fukui functions dependent on total electronic densities. As such, $f^{+}(r)=\rho_{N+1}(r)-\rho_{N}(r)$ and $f^{-}(r)=$ $\rho_{N(r)}-\rho_{N-1}(r)$, with $\rho_{N+1}(r), \rho_{N}(r)$ and $\rho_{N-1}(r)$ being the electronic densities corresponding to point $\mathbf{r}$ for a molecular system with $N_{+} 1, N$ and $N-1$ electrons. The initial definition $f^{+}(r=$ associates the reactivity to nucleophilic attack thus measuring the intramolecular reactivity occurring at site $r$ directed to the nucleophilic reagent. The second definition $f^{-}(r)$ associates the reactivity to electrophilic attack thereby measuring the intramolecular reactivity at $r$ towards the corresponding electrophilic reagent [13].

This study makes reference to Morell et al. [19-25] proposal on the local reactivity descriptor (LRD) they identified as the dual descriptor (DD) and defined as $f^{(2)}(r) \equiv \Delta f(r)$. Their findings demonstrated its mathematical definition as a derivative of Fukui function $f(r)^{14}$ relative to the electrons number $N$. The definition suggests $\Delta f_{k}>0$ shows a process driven by nucleophilic attack occurring at atom $k$ thus leaving the atom that acts of electrophilic species. A converse of this conclusion emerges when $\Delta f(r)<0$ indicating a process caused by electrophilic attacks on atom $k$ forcing it to act as nucleophilic species.

\section{SETTINGS AND COMPUTATIONAL METHODS}

Thisstudy adopts the previous work context as outlined in detail [5-12] using the Gaussian 09 series of programs to perform all the computations. Similarly, it implements density functional approaches guided by the Gaussian computational package [26]. Besides, the gradient technique was adopted to determine the equilibrium geometries that the studied molecules assume. The computation of analytical frequencies performed on stationary sites facilitated the determination of vibrational frequencies and force constants. The Def2SVP basis set to determine if they generated the true minima was applied for geometry optimization as well as the Def2TZVP set in calculating the electronic properties [27,28].

The procedure uses density functionals derived from latest Minnesota family to calculate the system properties and molecular structure. Specifically, the study uses density functional 
proven of generating satisfactory results in thermodynamic properties and fitting multiple structural systems [29]. They include M11 being a range-classified hybrid meta-GGA, [30] M11L a dual-range local meta-GGA, [31] MN12L a unified local meta-NGA, [32] MN12SX a rangesplit hybrid unified meta-NGA, [33] N12 a unified gradient approximation, [34] N12SX, a range-categorized hybrid unified gradient approximation, [33] SOGGA11 GGA density functional [35] and SOGGA11X a mixed GGA density functional [36]. The acronym GGA represents the comprehensive gradient approximation indicating the density functional dependent on alternating spins and reduced gradient. NGA is the unified gradient approximation upon which the functional relies upon the alternating spinning and reduced gradient densities. Further consideration is made on other density functionals including BLYP, [37,38] B3LYP, [38,39] PBE, [40,41] PBE0, [40-42] CAM-B3LYP, [43] LC-wPBE, [44-46] HSEh1PBE, [47-53] wB97, [54] wB97X, [54] wB97XD, [55] M05, [56] M052X, [57,58] M06, [58-59] M06L, [58,60] M062X [58,59] and M06HF [58,61]. A procedural reference was made from the SMD solvation model [62] in performing the IEF-PCM computations in all the calculations using water as the solvent.

\section{RESULTS AND DISCUSSION}

Initial pre-optimization was obtained on the Allose, Altrose, Arabinose, Galactose, Glucose, Mannose, Lyxose, Ribose, Talose and Xylose molecular structures. Pre-optimization targeted MOL structures to identify the conformers that are most stable using the Avogadro 1.2.0 program $[63,64]$. The processes applied random sampling integrated with particular molecular mechanics methods and examination of all torsional angles. Subsequent reoptimization was performed based on the structures assumed by produced conformers using the 24 density functionals. This involved the Def2SVP basis set as well as SMD solvation approach with water the solvent in all processes.

The calculation of the global electrophilicity $\omega$, electronegativity $\chi$, and global hardness $\eta$ for the systems seeks verifying the KID procedure for validity. Additional verifications are attained by calculating the electrodonating power $\omega^{-}$, electroaccepting power $\omega^{+}$and net electrophilicity $\Delta \omega^{ \pm}$.

The results of the calculations performed are captured in the Tables S1A through to S24A as Electronic Supplementary Information. The tables represent the values of $\mathrm{HOMO}$ orbital energies (eV), LUMO orbital energies (depicted in terms of eV), ionization potentials I (also in eV), electron affinities A (eV), global electronegativity depicted as $\chi$, global hardness represented as $\eta$, global electrophilicity $\omega$, electrodonating power $\omega^{-}$, electroaccepting power in $\omega^{+}$and net eletrophilicity represented as $\Delta \omega^{ \pm}$.The upper sections of each table represent the results obtained assuming verified validity of Koopmans in DFT - a reason for the subscript K. The lower segment shows results obtained by calculating vertical I as well as $A$.

This study uses designed descriptors to analyze results in verifying achievement of the "Koopmans in DFT" procedure. This is inspired by preceding works, $[65,66]$ to use descriptors in relating the results achieved with those obtained through calculating $\mathrm{HOMO}$ and LUMO energies using vertical I and A using a $\triangle$ SCF procedure [5]. This study does not aim performing a gapfitting by minimizing the descriptor using the optimal range-separation parameter $\gamma$. Instead, it aims checking if the density functionals used comply with the "Koopmans in DFT" procedure with some possessing the fixed range-separation parameter $\gamma$. The designed descriptors avoid using range-separation parameter $\gamma$. In addition, $A$ is regarded the minus energy in LUMO derived from a neutral system rather than being the minus energy representing $\mathrm{HOMO}$ in a $\mathrm{N}+1$ electron system as earlier mentioned $[65,66]$.

The initial set of three descriptors demonstrate simple fulfillment of the "Koopmans in DFT" procedure by associating the individual description of the HOMO-LUMO gap derived from $\varepsilon_{\mathrm{H}}$ with -land $\varepsilon_{\mathrm{L}}$ with $-\mathrm{A}$ links. This fulfillment is captured as $J_{I}=\left|\epsilon_{H}+E_{g s}(N-1)-E_{g s}(N)\right|$, $J_{A}=\left|\epsilon_{L}+E_{g s}(N)-E_{g s}(N+1)\right|$ and $J_{H L}=$ $\sqrt{J_{I}^{2}+J_{A}^{2}}$.

The next set involves four descriptors analyzing how well the density functions predict the global hardness, global electronegativity and electrophilicity and their combined form in Conceptual DFT descriptors in examining orbital energies for both HOMO and LUMO alongside vertical $\mathrm{I}$ and $\mathrm{A}$. This is represented as $J_{\chi}=$ $\left|\chi-\chi_{K}\right|, \quad J_{\eta}=\left|\eta-\eta_{K}\right|, \quad J_{\omega}=\left|\omega-\omega_{K}\right|$ and $J_{D 1}=\sqrt{J_{\chi}^{2}+J_{\eta}^{2}+J_{\omega}^{2}}$. D1 being the initial group of Conceptual DFT descriptors.

The final set of four descriptors sought verifying how good the density functional were in 
predicting the electrodonating powers $\omega^{-}$, electroaccepting powers $\omega^{+}$and net eletrophilicity $\Delta \omega^{ \pm}$and the combined case for Conceptual DFT descriptors. Again, the HOMO and LUMO energies were considered for the vertical $I$ and $A$ shown as $J_{\omega^{+}}=\left|\omega^{+}-\omega_{K}^{+}\right|$, $J_{\omega^{-}}=\left|\omega^{-}-\omega_{K}^{-}\right| \quad, \quad J_{\Delta \omega^{ \pm}}=\left|\Delta \omega^{ \pm}-\Delta \omega_{K}^{ \pm}\right| \quad$ and $J_{D 2}=\sqrt{J_{\omega^{+}}^{2}+J_{\omega^{-}}^{2}+J_{\Delta \omega^{ \pm}}^{2}}$, with D2 representing the first group of conceptual DFT descriptors.

Tables S1B to S24B display the results obtained of $\mathrm{J}_{1}, \mathrm{~J}_{\mathrm{A}}, \mathrm{J}_{\mathrm{HL}}, \mathrm{J}_{\chi}, \mathrm{J}_{\eta}, \mathrm{J}_{\omega}, \mathrm{J}_{\mathrm{D} 1}, J_{\omega^{+}}, J_{\omega^{-}}, J_{\Delta \omega^{ \pm}}$and $\mathrm{J}_{\mathrm{D} 2}$ for both hexoses and pentoses systems. The results displayed in Table 1 involve compiled average values for all density functional on the entire set of hexoses and pentoses.

Table 1 illustrates the "Koopmans in DFT" procedure upholding great accuracy noted in MN12SX a range-separated hybrid meta-NGA, N12SX a range-separated hybrid NGA and HSEh1PBE density functionals. The table shows $J_{I}, J_{A}, J_{H L}$ having non-zero values. Indeed, the obtained values compare favorably with the Lima et al. [66] findings, where the quantities revealed generated minima by using the parameter $\gamma$ enforcing such behavior.

The results obtained interestingly showed the same density functionals obeying the "Koopmans in DFT" procedure in other set of descriptors. Specifically, the fulfillment of the procedure was noted for $J_{\chi}, J_{\eta}, J_{\omega}, J_{D 1}$, alongside with $J_{\omega-}, J_{\omega+}$, $J_{\omega \pm}$ and $J_{\mathrm{D} 2}$. These findings are important, since they affirmed that the study could not depend solely on $J_{l}, J_{A}$, and $J_{H L}$. Taking a case $J_{\chi}$ for demonstrate near zero values for all calculations made on all density functional. A cross-sectional analysis shows only HSEh1PBE, MN12SX and N12SX revealed a similar behavior. This implies the possibility of accidental cancellation of errors.

Results attained on the hybrid-GGA (SOGGA11X) and GGA (SOGGA11) showed failed fulfillment of the "Koopmans in DFT" procedure. A similar conclusion was reached on M11L, N12 and MN12L density functionals. While the range-separated hybrid NGA and range-separated hybrid meta-NGA showed usefulness in calculating Conceptual DFT descriptors, it is not the same for the rangeseparated hybrid GGA (M11). A reflection on Table S17A illustrates its inadequacy in describing the LUMO energy. This caused negative values in $\mathrm{A}$ thereby contradicting $\triangle \mathrm{SCF}$ results.
The objective of this work captured in the introduction involves finding the best description for the glycating power of carbohydrates based upon accurate calculations on the Conceptual DFT descriptors. In light of this, we plotted various Conceptual DFT descriptors values as captured on tables S1A- S24A against the reaction constants of monosaccharides while reacting with pyridoxamine $\left(k_{3}\right)$ from the Adrover et al study [4]. Performing a visual inspection of the plots show the best correlations occurring between global electrophilicitywand $k_{3}$. This mandates performing a linear regression analysis on the results obtained defined as $G P=a \omega+b$ with GP representing $k_{3}$, a being the slope and $b$ interception point in the linear correlation. Table 2 shows the results obtained from the linear analysis on the 24 density functionals.

The results show high MCC figures for most functionals, though different for N12, N12SX, and SOGGA11 and SOGGA11X. A similar magnitude is noted for typical error values, again different for N12, N12SX, SOGGA11 and SOGGA11X whose values 2 to 3 times greater. The MAD display disparate values for the density functional with M06 and MN12SX having the best results of below 0.6.

It is equally important to monitor the inclusion of local descriptors in the above linear correlations. This inclusion would serve checking if enhanced description of the glycating power is possible, specifically by obtaining lower MAD.

The use of condensed Fukui functions could also help to identify the reactivity site that each atom occupies in the studied molecules. The following condensed Fukui functions were applied: $f_{k}^{+}=q_{k}(N+1)-q_{k}(N)$ showing the nucleophilic attack, $f_{k}^{-}=q_{k}(N)-q_{k}(N-1)$ testing electrophilic attack and $f_{k}^{0}=\left[q_{k}(N+1)-q_{k}(N)\right] / 2$ indicating radical attack, with $q_{k}$ showing the atom $\mathrm{k}$ gross charge in the studied molecules. It is worth mentioning that AOMix program for molecular analysis $[67,68]$ was applied to calculate the condensed Fukui functions.

The condensed dual descriptor derived from the condensed Fukui functions is defined as $\Delta \mathrm{f}_{\mathrm{k}}=$ $\mathrm{f}_{\mathrm{k}}^{+}-\mathrm{f}_{\mathrm{k}}^{-}[19,20]$. This definition reveals that the dual descriptors sign is critical in characterizing the site reactivity in a molecule, either showing an electrophilic or nucleophilic attack. This suggests that $\Delta \mathrm{f}_{\mathrm{k}}<0$ shows electrophilic attack while that $\Delta \mathrm{f}_{\mathrm{k}}<0$ a nucleophilic attack $[19,20,69]$. 
Frau and Glossman-Mitnik; BJAST, 21(1): 1-12, 2017; Article no.BJAST.32795

Table 1. Average descriptors $\mathrm{J}_{1}, \mathrm{~J}_{\mathrm{A}}, \mathrm{J}_{\mathrm{HL}}, \mathrm{J}_{\chi}, \mathrm{J}_{\eta}, \mathrm{J}_{\omega}, \mathrm{J}_{\mathrm{D} 1}, \mathrm{~J}_{\omega-1}, \mathrm{~J}_{\omega+}, \mathrm{J}_{\omega+}$ and $\mathrm{J}_{\mathrm{D} 2}$ for the Allose, Altrose, Arabinose, Galactose, Glucose, Gulose, Lyxose, Mannose, Ribose, Talose and Xylose molecules calculated with the twenty-four density functionals and the Def2TZVP basis set using water as solvent simulated with the SMD parameterization of the IEF-PCM model

\begin{tabular}{|c|c|c|c|c|c|c|c|c|c|c|c|}
\hline & $\mathbf{J}_{\mathbf{I}}$ & $\mathbf{J}_{\mathrm{A}}$ & $J_{\mathrm{HL}}$ & $J_{x}$ & $J_{n}$ & $J_{\omega}$ & $J_{D 1}$ & $J_{\omega}$ & $\mathbf{J}_{\omega+}$ & $J_{\omega \pm}$ & $\mathbf{J}_{\mathrm{D} 2}$ \\
\hline BLYP & 0.439 & 0.697 & 0.826 & 0.129 & 1.136 & 0.623 & 1.303 & 1.240 & 1.112 & 2.352 & 2.882 \\
\hline B3LYP & 0.215 & 0.272 & 0.349 & 0.039 & 0.487 & 0.159 & 0.514 & 0.303 & 0.274 & 0.577 & 0.708 \\
\hline PBE & 0.434 & 0.693 & 0.820 & 0.130 & 1.128 & 0.662 & 1.315 & 1.319 & 1.189 & 2.508 & 3.073 \\
\hline PBE0 & 0.394 & 0.547 & 0.675 & 0.078 & 0.941 & 0.296 & 0.990 & 0.571 & 0.495 & 1.066 & 1.306 \\
\hline CAM-B3LYP & 1.508 & 1.812 & 2.358 & 0.152 & 3.320 & 0.700 & 3.397 & 1.268 & 1.116 & 2.383 & 2.921 \\
\hline LC-wPBE & 2.618 & 3.167 & 4.110 & 0.275 & 5.785 & 0.994 & 5.877 & 1.765 & 1.490 & 3.255 & 3.991 \\
\hline HSEh1PBE & 0.022 & 0.157 & 0.160 & 0.070 & 0.177 & 0.104 & 0.218 & 0.233 & 0.163 & 0.396 & 0.358 \\
\hline wB97 & 2.599 & 3.114 & 4.058 & 0.258 & 5.713 & 0.916 & 5.793 & 1.604 & 1.347 & 2.951 & 3.619 \\
\hline wB97X & 2.425 & 2.860 & 3.750 & 0.217 & 5.284 & 0.874 & 5.361 & 4.492 & 0.191 & 2.837 & 5.318 \\
\hline wB97XD & 2.059 & 2.376 & 3.144 & 0.158 & 4.435 & 0.782 & 4.506 & 2.868 & 1.208 & 2.575 & 4.042 \\
\hline M05 & 0.405 & 0.629 & 0.749 & 0.112 & 1.034 & 0.311 & 1.086 & 0.614 & 0.502 & 1.116 & 1.369 \\
\hline M052X & 1.472 & 1.740 & 2.280 & 0.134 & 3.212 & 0.668 & 3.284 & 1.201 & 1.068 & 2.269 & 2.781 \\
\hline M06 & 0.384 & 0.515 & 0.644 & 0.071 & 0.900 & 0.267 & 0.941 & 0.511 & 0.446 & 0.957 & 1.173 \\
\hline M06L & 0.362 & 0.472 & 0.596 & 0.055 & 0.834 & 0.344 & 0.904 & 0.663 & 0.608 & 1.271 & 1.337 \\
\hline M062X & 1.401 & 1.608 & 2.135 & 0.106 & 3.010 & 2.795 & 4.110 & 1.091 & 0.987 & 2.078 & 2.546 \\
\hline M06HF & 3.144 & 3.334 & 4.584 & 0.111 & 6.478 & 0.955 & 6.549 & 1.553 & 1.458 & 3.011 & 3.688 \\
\hline M11 & 2.462 & 2.822 & 3.746 & 0.180 & 5.284 & 0.906 & 5.365 & 1.573 & 1.393 & 2.965 & 3.634 \\
\hline M11L & 0.503 & 0.523 & 0.544 & 0.556 & 0.633 & 0.633 & 0.711 & 0.719 & 0.807 & 0.992 & 1.092 \\
\hline MN12L & 0.320 & 0.254 & 0.411 & 0.045 & 0.573 & 0.141 & 0.593 & 0.230 & 0.263 & 0.494 & 0.606 \\
\hline MN12SX & 0.051 & 0.178 & 0.189 & 0.067 & 0.229 & 0.109 & 0.305 & 0.235 & 0.171 & 0.405 & 0.423 \\
\hline N12 & 0.394 & 0.690 & 0.795 & 0.148 & 1.084 & 0.550 & 1.352 & 1.105 & 0.958 & 2.063 & 1.993 \\
\hline N12SX & 0.009 & 0.140 & 0.140 & 0.066 & 0.149 & 0.091 & 0.215 & 0.206 & 0.140 & 0.346 & 0.329 \\
\hline SOGGA11 & 0.451 & 0.761 & 0.887 & 0.155 & 1.212 & 0.699 & 1.510 & 1.400 & 1.245 & 2.645 & 2.409 \\
\hline SOGGA11X & 1.116 & 1.215 & 1.652 & 0.054 & 2.331 & 0.506 & 2.858 & 0.891 & 0.842 & 1.733 & 3.113 \\
\hline
\end{tabular}


Table 2. Linear regression parameters: Slope a, Interception b, multiple correlation coefficient MCC, Typical Error TE and Mean Absolute Deviation MAD for the Allose, Altrose, Arabinose,

Galactose, Glucose, Gulose, Lyxose, Mannose, Ribose, Talose and Xylose molecules calculated with the twenty-four density functionals and the Def2TZVP basis set using water as solvent simulated with the SMD parameterization of the IEF-PCM model

\begin{tabular}{llllll}
\hline & $\mathbf{a}$ & $\mathbf{b}$ & MCC & TE & MAD \\
\hline BLYP & 55.5758 & -82.4942 & 0.9498 & 0.9822 & 0.7899 \\
B3LYP & 65.6780 & -102.3943 & 0.9621 & 0.8557 & 0.6722 \\
PBE & 45.1506 & -65.9714 & 0.9337 & 1.1242 & 0.8727 \\
PBE0 & 60.7400 & -93.1869 & 0.9491 & 0.9886 & 0.8065 \\
CAM-B3LYP & 61.1536 & -96.6709 & 0.9489 & 0.9905 & 0.8188 \\
LC-wPBE & 58.5259 & -97.2502 & 0.9183 & 1.2429 & 1.0103 \\
HSEh1PBE & 60.2928 & -92.7533 & 0.9507 & 0.9735 & 0.7872 \\
wB97 & 62.6414 & -96.7526 & 0.9328 & 1.1314 & 0.9622 \\
wB97X & 65.0791 & -100.5809 & 0.9374 & 1.0933 & 0.9264 \\
wB97XD & 68.1462 & -103.7450 & 0.9536 & 0.9447 & 0.7787 \\
M05 & 64.3896 & -91.3532 & 0.9483 & 0.9968 & 0.7813 \\
M052X & 72.4292 & -117.3654 & 0.9539 & 0.9243 & 0.7444 \\
M06 & 66.3025 & -100.5016 & 0.9646 & 0.8283 & 0.5917 \\
M06L & 70.5378 & -98.7481 & 0.9446 & 1.0303 & 0.6763 \\
M062X & 79.5595 & -126.0879 & 0.9545 & 0.9362 & 0.7588 \\
M06HF & 79.7895 & -137.5252 & 0.9497 & 0.9834 & 0.6850 \\
M11 & 65.2613 & -107.5261 & 0.9288 & 1.1637 & 0.9199 \\
M11L & 63.5265 & -95.4417 & 0.9359 & 1.1059 & 0.8112 \\
MN12L & 82.8255 & -109.8228 & 0.9362 & 1.1033 & 0.7651 \\
MN12SX & 87.5200 & -134.3312 & 0.9532 & 0.9496 & 0.5840 \\
N12 & 38.4658 & -46.5835 & 0.8808 & 1.4863 & 1.2332 \\
N12SX & 44.9554 & -64.3326 & 0.6430 & 2.4044 & 1.4311 \\
SOGGA11 & -4.7053 & 16.6130 & 0.1009 & 3.1233 & 2.1970 \\
SOGGA11X & -21.8945 & 46.2125 & 0.7377 & 2.1195 & 1.7211 \\
\hline
\end{tabular}

Table 3. Condensed dual descriptors $\Delta f_{k}$ over the carbonyl $\mathrm{C}$ atoms of theAllose, Altrose,

Arabinose, Galactose, Glucose, Gulose, Lyxose, Mannose, Ribose, Talose and Xylose molecules calculated with the M06 and MN12SX density functionals and the Def2TZVP basis set using water as solvent simulated with the SMD parameterization of the IEF-PCM model

\begin{tabular}{lll}
\hline & M06 & MN12SX \\
\hline Allose & 0.6172 & 0.6154 \\
Altrose & 0.6077 & 0.5964 \\
Arabinose & 0.5558 & 0.5554 \\
Galactose & 0.5691 & 0.6171 \\
Glucose & 0.6140 & 0.6117 \\
Gulose & 0.6278 & 0.6292 \\
Lyxose & 0.5938 & 0.6027 \\
Mannose & 0.6005 & 0.5957 \\
Ribose & 0.5923 & 0.5835 \\
Talose & 0.5960 & 0.5893 \\
Xylose & 0.6099 & 0.6089 \\
\hline
\end{tabular}

The condensed dual descriptor $\Delta \mathrm{f}_{\mathrm{k}}$ was again computed using the M06 and MN12SX density functionals over carbonyl $\mathrm{C}$ atoms on Allose, Altrose, Arabinose, Galactose, Glucose, Gulose, Lyxose, Mannose, Ribose, Talose and Xylose molecules with the Def2TZVP basis set and water serving as the solvent simulated using the SMD parametrization of the IEF-PCM model. Table 3 displays the results obtained.

From the results displayed, a new linear regression analysis was necessary to improve the prediction of the glycating power. A new equation was used $G P=a_{1} \omega+a_{2} \Delta f_{k}+b$. GP represents $k_{3}$ while $a_{1}, a_{2}$ and $b$ illustrated the correlation parameters. The results facilitated an input to analyze M06 and MN12SX further as shown in Table 4.

The values for MAD shows an improvement for the MN12SX density functional. From this change, the following formula can be applied in predicting the glycating power (GP) of carbohydrates with simple structures $G P=$ $94.6491 \omega-29.5963 \Delta f_{k}-128.2751$, with $\omega$ being the global electrophilicity and $\Delta f_{k}$ representing the condensed dual descriptor 
Table 4. Linear regression parameters $a_{1}$, a2, and b, Multiple Correlation Coefficient MCC, Typical Error TE and Mean Absolute Deviation MAD for the Allose, Altrose, Arabinose, Galactose, Glucose, Gulose, Lyxose, Mannose, Ribose, Talose and Xylose molecules calculated with the M06 and MN12SX density functionals and the Def2TZVP basis set using water as solvent simulated with the SMD parametrization of the IEF-PCM model

\begin{tabular}{lllllll}
\hline & $\mathbf{a}_{1}$ & $\mathbf{a}_{2}$ & $\mathbf{b}$ & $\mathbf{M C C}$ & TE & MAD \\
\hline M06 & 68.7579 & -8.9367 & -99.226 & 0.966 & 0.8613 & 0.589 \\
MN12SX & 94.6491 & -29.596 & -128.28 & 0.9706 & 0.8014 & 0.5212 \\
\hline
\end{tabular}

whose values are computed using MN12SX with the Def2TZVP basis set with water serving as the solvent simulated using the SMD parametrization on IEF-PCM model.

\section{CONCLUSIONS}

This study investigated compliance with the "Koopmans in DFT" procedure in the Minnesota family of density functionals - (M11, M11L, MN12L, MN12SX, N12, N12SX, SOGGA11 and SOGGA11X)and 16 usual density functionals by comparing their derived values for $\mathrm{HOMO}$ and LUMO energies with those generated using the $\triangle$ SCF approach. The results demonstrate that N12SX a range-separated hybrid NGA, HSEh11PBE and MN12SX a range-separated hybrid meta-NGA functional are the best for achieving the study objective. They demonstrate as a good alternative in their use than density functionals that feature a behavior tuned using a gap-fitting procedure. They represent applicability in describing chemical reactivity of large-sized molecules.

The results obtained for this work shows the ability to predict the interaction sites of simple carbohydrates by applying DFT-based descriptors of chemical reactivity. Such include global electronegativity, global hardness, global electrophilicity, electrodonating powers, electroaccepting powers, net electrophilicity, Fukui function and condensed dual descriptor. Their use in characterizing and describing preferred reactivity sites generated a firm explanation on the molecule reactivity.

The results show a good relationship existing between glycating power GP in carbohydrates of simple structures and several Conceptual DFT descriptors. This is demonstrated by computations applying MN12SX density functional and the Def2TZVP basis set where water acts as the solvent simulated through SMD parametrization guided by the IEF-PCM model. The results reveal that
MN12SX/Def2TZVP/SMD-Water model best accomplish the "Koopmans in DFT" procedure and successfully predicts glycating power in simple carbohydrates. This confirms its applicability in studying the behavior of molecules with carbonyl $\mathrm{C}$ atoms involved in the Maillard reaction for specific complex carbohydrates.

The introduction section captures the basis of this study as a part of ongoing project to examine glycation occurring in amino acids and perhaps proteins from reducing carbonyls in the formation of AGEs inhibitors. This study focuses on monosaccharides assuming hexoses and pentoses in a comparison with results obtained from other experimental studies. Subsequent studying of ketoses would be interesting especially examining the fructose involved in Maillard reaction. The findings obtained in this study do not possibly allow speculations about the comparison of the GP of fructose with aldoses results. However, the findings can foresight that since fructose assumes a greater open-chain form than glucose, it will probably have a higher GP.

\section{ACKNOWLEDGEMENTS}

This work has been partially supported by CIMAV, SC and Consejo Nacional de Ciencia y Tecnología (CONACYT, Mexico) through Grant 219566/2014 for Basic Science Research and Grant 265217/2016 for a Foreign Sabbatical Leave. Daniel Glossman-Mitnik conducted this work while a Sabbatical Fellow at the University of the Balearic Islands from which support is gratefully acknowledged. This work was cofunded by the Ministerio de Economía y Competitividad (MINECO) and the European Fund for Regional Development (FEDER) (CTQ2014-55835-R).

\section{SUPPLEMENTARY DATA}

Supplementary data related to this article can be found at the online version of this article. 
Link:https://drive.google.com/file/d/OBw Cay1wxU3Rm91Z1hFVEpNNGs/view?usp=sharin g

\section{COMPETING INTERESTS}

Authors have declared that no competing interests exist.

\section{REFERENCES}

1. Ahmed N. Advanced glycation endproducts - Role in pathology of diabetic complications. Diabetes Res Clin Pract. 2005;67:3-21.

2. Li J, Liu D, Sun L, Lu Y, Zhang Z. Advanced glycation end products and neurodegenerative diseases: Mechanisms and perspective. J Neurol Sci. 2012;317 (1-2):1-5.

3. Peyroux J, Sternberg M. Advanced glycation endproducts (AGEs): Pharmacological inhibition in diabetes. Pathol Biol. 2006;54:405-419.

4. Adrover $M$, Vilanova $B$, Muñoz $F$, Donoso J. Pyridoxamine, a scavenger agent of carbohydrates. Int J ChemKinet. 2007;39 (3):154-167.

5. Frau J, Glossman-Mitnik D. A conceptual DFT study of the molecular properties of glycating carbonyl compounds. Chem Cent J. 2017;11(1):8.

6. Glossman-Mitnik D. A comparison of the chemical reactivity of naringenin calculated with the M06 family of density functionals. Chem Cent J. 2013;7:155-161.

7. Martínez-Araya JI, Salgado-Morán, Glossman-Mitnik D. Computational nanochemistry report on the oxicams Conceptual DFT and chemical reactivity. J Phys Chem B. 2013;117(21):6639-6651.

8. Glossman-Mitnik D. Computational nanochemistry study of the chemical reactivity properties of the Rhodamine $B$ molecule. Procedia Comput Sci. 2013; 18:816-825.

9. Martínez-Araya JI, Salgado-Morán G, Glossman-Mitnik D. Computational nutraceutics: Chemical reactivity properties of the flavonoid naringin by means of conceptual. DFT. J Chem. 2013;850270.

10. Glossman-Mitnik D. Chemical reactivity theory within DFT applied to the study of the prunin flavonoid. Eurlnt $\mathrm{J}$ Sci and Technol. 2014;3(9):195-207.

11. Glossman-Mitnik D. Computational chemistry of natural products: A comparison of the chemical reactivity of isonaringin calculated with the M06 family of density functionals. J Mol Mod. 2014; 20:1-7.

12. Frau J, Muñoz F, Glossman-Mitnik D. A molecular electron density theory study of the chemical reactivity of cis- and trans-resveratrol. Molecules. 2016;21(12): 1650.

13. Parr RG, Yang W. Density functional approach to the frontier-electron theory of chemical reactivity. J Am Chem Soc. 1984; 106:4049-4050.

14. Geerlings P, De Proft F, Langenaeker W. Conceptual density functional theory. Chem Rev. 2003;103:1793-1873.

15. Parr RG, Szentpaly L, Liu S. Electrophilicity index. J Am Chem Soc. 1999;121:1922-1924.

16. Gázquez JL, Cedillo A, Vela A. Electrodonating and electroaccepting powers. J Phys Chem A. 2007;111(10): 1966-1970.

17. Chattaraj PK, Chakraborty A, Giri S. Net electrophilicity. J Phys Chem A. 2009; 113(37):10068-10074.

18. Ayers P. The dependence on and continuity of the energy and other molecular properties with respect to the number of electrons. J Math Chem. 2008; 43:285-303.

19. Morell C, Grand A, Toro-Labbé A. New dual descriptor for chemical reactivity. J Phys Chem A. 2005;109:205212.

20. Morell C, Grand A, Toro-Labbé A. Theoretical support for using the $\Delta f(r)$ descriptor. Chem Phys Lett. 2006;425: 342-346.

21. Cárdenas C, Rabi N, Ayers P, Morell C, Jaramillo P, Fuentealba P. Chemical reactivity descriptors for ambiphilic reagents: Dual descriptor, local hypersoftness, and electrostatic potential, J Phys Chem A. 2009;113:8660-8667.

22. Toro-Labbé A, editor. Theoretical aspects of chemical reactivity. Elsevier Science (Amsterdam); 2007.

23. Ayers $P$, Morell $C$, De Proft $F$, Geerlings $P$. Understanding the woodward-hoffmann rules by using changes in electron density. Chem Eur J. 2007;13:8240-8247.

24. Morell C, Ayers P, Grand A, GutiérrezOliva S, Toro-Labbé A. Rationalization of the Diels-Alder reactions through the use of the dual reactivity descriptor $\Delta f(r)$. 
Phys Chem Chem Phys. 2008;10:72397246.

25. Morell C, Hocquet A, Grand A, JamartGrégoire B. A conceptual DFT study of hydrazino peptides: Assessment of the nucleophilicity of the nitrogen atoms by means of the dual descriptor $\Delta f(r)$. J MoIStruc THEOCHEM. 2008;849:46-51.

26. Gaussian, Revision E, Frisch MJ, Trucks GW, Schlegel HB, Scuseria GE, Robb MA, Cheeseman JR, et al. Gaussian, Inc., Wallingford CT; 2016.

27. Weigend F, Ahlrichs R. Balanced basis sets of split valence, triple zeta valence and quadruple zeta valence quality for $\mathrm{H}$ to $\mathrm{Rn}$ : Design and assessment of accuracy. Phys ChemChem Phys. 2005;7:32973305.

28. Weigend F. Accurate Coulomb-fitting basis sets for $\mathrm{H}$ to $\mathrm{R}$, Phys ChemChem Phys. 2006; 8:1057-1065.

29. Peverati R, Truhlar DG. Quest for a universal density functional: The accuracy of density functionals across a broad spectrum of databases in chemistry and physics. Philos Trans A Math Phys Eng Sci. 2014;372(2011):20120476.

30. Peverati R, Truhlar DG. Improving the accuracy of hybrid meta-GGA density functionals by range separation. J Phys Chem Lett. 2011;2(21):2810-2817.

31. Peverati R, Truhlar DG. M11-L: A local density functional that provides improved accuracy for electronic structure calculations in chemistry and physics. J Phys Chem Lett. 2012;3(1):117-124.

32. Peverati R, Truhlar DG. An improved and broadly accurate local approximation to the exchange-correlation density functional: The MN12-L functional for electronic structure calculations in chemistry and physics. Phys ChemChem Phys. 2012; 14(38):13171-13174.

33. Peverati R, Truhlar DG. Screenedexchange density functionals with broad accuracy for chemistry and solid-state physics, Phys ChemChem Phys. 2012; 14(47):16187-16191.

34. Peverati $R$, Truhlar DG. Exchangecorrelation functional with good accuracy for both structural and energetic properties while depending only on the density and its gradient. J Chem Theor Comput. 2012; 8(7):2310-2319.

35. Peverati R, Zhao $Y$, Truhlar DG. Generalized gradient approximation that recovers the second-order density-gradient expansion with optimized across-the-board Performance. J Phys Chem Lett. 2011; 2(16):1991-1997.

36. Peverati R, Truhlar DG. Communication: A global hybrid generalized gradient approximation to the exchange-correlation functional that satisfies the second-order density-gradient constraint and has broad applicability in chemistry. J Chem Phys. 2011;135(19):191102.

37. Becke A. Density-functional exchangeenergy approximation with correct asymptotic-behavior. Phys Rev A. 1988; 38:3098-3100.

38. Lee C, Yang W, Parr RG. Development of the Colle-Salvetti correlationenergy formula into a functional of the electron density. Phys Rev B. 1988; 37:785-789.

39. Becke A. Density-functional thermochemistry. III. The role of exact exchange. J Chem Phys. 1993;98:56485652.

40. Perdew J, Burke K, Ersernhof $M$. Generalized gradient approximation made simple. Phys Rev Lett. 1996;77:38653868.

41. Perdew J, Burke K, Ersernhof M. Erratum: Generalized gradient approximation made simple. Phys Rev Lett. 1997;78:1396.

42. Adamo C, Barone V. Toward reliable density functional methods without adjustable parameters: The PBE0 model. Chem Phys. 1999;110:6158-6169.

43. Yanai T, Tew DP, Handy NC. A new hybrid exchange-correlation functional using the Coulomb-attenuating method (CAMB3LYP). Chem Phys Lett. 2004;393(13):51-57.

44. Vydrov OA, Scuseria GE. Assessment of a long-range corrected hybrid functional. J Chem Phys. 2006;125(23):234109.

45. Vydrov OA, Heyd J, Krukau AV, Scuseria GE. Importance of short-range versus long-range Hartree-Fock exchange for the performance of hybrid density functionals. J Chem Phys. 2006; 12(7):074106.

46. Vydrov OA, Scuseria GE, Perdew JP. Tests of functionals for systems with fractional electron number. J Chem Phys. 2007;126(15):154109.

47. Heyd J, Scuseria GE. Efficient hybrid density functional calculations in solids: Assessment of the Heyd-Scuseria- 
Ernzerhof screened Coulomb hybrid functional. J Chem Phys. 2004;121(3): 1187-1192.

48. Heyd J, Peralta JE, Scuseria GE, Martin RK. Energy band gaps and lattice parameters evaluated with the HeydScuseria-Ernzerh of screened hybrid functional. J Chem Phys. 2005;123(17): 174101.

49. Heyd J, Scuseria GE, Ernzerhof $M$. Erratum: Hybrid functionals based on a screened coulomb potential. J. Chem. Phys. 2003;118:8207. J Chem Phys. 2006;124(21):219906.

50. Izmaylov AF, Scuseria GE, Frisch MJ. Efficient evaluation of short-range HartreeFock exchange in large molecules and periodic systems. J Chem Phys. 2006; 125(10):104103.

51. Krukau AV, Vydrov OA, Izmaylov AF, Scuseria GE. Influence of the exchange screening parameter on the performance of screened hybrid functionals. J Chem Phys. 2006;125(22):224106.

52. Henderson TM, Izmaylov AF, Scalmani G, Scuseria GE. Can short-range hybrids describe long-range-dependent properties?, J Chem Phys. 2009;131 (4):044108.

53. Chai J, Head-Gordon M. Systematic optimization of long-range corrected hybrid density functionals. J Chem Phys. 2008; 128-084106.

54. Chai J, Head-Gordon M. Long-range corrected hybrid density functionals with damped atom-atom dispersion corrections, Phys ChemChem Phys. 2008;10:66156620.

55. Zhao $Y$, Schultz NE, Truhlar DG. Exchange-correlation functional with broad accuracy for metallic and nonmetallic compounds, kinetics, and noncovalent interactions. J Chem Phys. 2005;123(16): 161103.

56. Heyd J, Scuseria GE. Assessment and validation of a screened coulomb hybrid density functional. J Chem Phys. 2004; 120(16):7274-7280.

57. Zhao Y, Schultz NE, Truhlar DG. Design of density functionals by combining the method of constraint satisfaction with parametrization for thermochemistry, thermochemical kinetics, and noncovalent interactions. J Chem Theor Comput. 2006; 2(2):364-382.
58. Zhao Y, Truhlar DG. Density functionals with broad applicability in chemistry. AccChem Res. 2008;41(2):157-167.

59. Zhao Y, Truhlar DG. The M06 suite of density functionals for main group thermochemistry, thermochemical kinetics, noncovalent interactions, excited states, and transition elements: Two new functionals and systematic testing of four M06-class functionals and 12 other functionals. Theor Chem Acc. 2008;120: 215-241.

60. Zhao Y, Truhlar DG. A new local density functional for main-group thermochemistry, transition metal bonding, thermochemical kinetics, and noncovalent interactions. J Chem Phys. 2006;125(19):194101.

61. Zhao Y, Truhlar DG. Density functional for spectroscopy: No long-range selfinteraction error, good performance for Rydberg and charge-transfer states, and better performance on average than B3LYP for ground states. J Phys Chem A. 2006;110(49):13126-13130.

62. Marenich A, Cramer C, Truhlar DG. Universal solvation model based on solute electron density and a continuum model of the solvent defined by the bulk dielectric constant and atomic surface tensions. J Phys Chem B. 2009;113:63786396.

63. Avogadro: An open-source molecular builder and visualization tool - Version 1.2.0; 2016.

Available:http://avogadro.openmolecules.n $\underline{\text { et }}$

64. Kronik L, Stein T, Refaely-Abramson $S$, Baer R. Excitation gaps of finitesized systems from optimally tuned range-separated hybrid functionals. J Chem Theor Comput. 2012;8(5):15151531.

65. Lima IT, Prado ADS, Martins JBL, de Oliveira Neto PH, Ceschin AM, da Cunha WF, da Silva Filho DA. Improving the description of the optical properties of carotenoids by tuning the long-range corrected functionals. J Phys Chem A. 2016;120(27):4944-4950.

66. Gorelsky S. AOMix Program for molecular orbital analysis - version 6.5, University of Ottawa, Ottawa, Canada; 2011.

67. Gorelsky S, Lever A. Electronic structure and spectra of ruthenium diimine complexes by Density Functional Theory 
and INDO/S - Comparison of the two methods. J Organomet Chem. 2001; 635(1-2):187-196.

68. Gázquez JL. Chemical reactivity concepts in density functional theory. In: Chattaraj PK, editor. Chemical reactivity theory: A density functional view. Boca Raton, Fl:
CRC Press - Taylor \& Francis Group; 2009.

69. Hanwell M, Curtis DE, Lonie DC, Vandermeersch T, Zurek E, Hutchison G. Avogadro: An advanced semantic chemical editor, visualization, and analysis platform. J Cheminf. 2012;4:17.

(c) 2017 Frau and Glossman-Mitnik; This is an Open Access article distributed under the terms of the Creative Commons Attribution License (http://creativecommons.org/licenses/by/4.0), which permits unrestricted use, distribution, and reproduction in any medium, provided the original work is properly cited.

Peer-review history:

The peer review history for this paper can be accessed here: http://sciencedomain.org/review-history/19110 